\title{
Relación entre la dimensión afectiva y el aprendizaje de las matemáticas
}

\author{
Relationship between Affective Dimension and Math Learning
}

\author{
Ronny Gamboa Araya' \\ Universidad Nacional \\ Heredia, Costa Rica \\ rgamboa@una.cr
}

Recibido11 de setiembre de 2013 • Corregido 21 de marzo de 2014 • Aceptado 2 de abril de 2014

Resumen. Las matemáticas se han convertido, para un número importante de estudiantes, en un obstáculo para el logro de sus objetivos escolares, de manera que ha trascendido la parte académica y se ha establecido como un impedimento cognitivo y emocional. Lo que el estudiantado siente, percibe, cree y su actuación influye directamente en esto. Aunado a lo anterior, lo que el sujeto docente siente, percibe, sus expectativas, creencias y actitudes respecto a la disciplina, también juegan un importante papel en el tipo de enseñanza que realiza y la dimensión afectiva de sus estudiantes. El presente artículo tiene como propósito abordar, basado en aspectos teóricos de varios autores, algunos elementos de la dimensión afectiva $y$, a partir de ellos, mostrar elementos concernientes tanto a docentes como a estudiantes y su relación con la enseñanza y aprendizaje de la matemática. Se obtuvo que el papel de la dimensión afectiva en el aprendizaje de las matemáticas debe ser un elemento abordado por la educación matemática como un medio para comprender este proceso desde la perspectiva de los actores relacionados con él, estudiantes y profesorado, así como para lograr un cambio en dicha disciplina a partir del mejoramiento de las creencias y actitudes del alumnado y personal docente hacia esta área del conocimiento.

Palabras clave. Actitudes, creencias, estudiantado, profesorado, enseñanza, aprendizaje, matemáticas.

Abstract. Math has become an obstacle to achieve educational goals for a large number of students; thus it has transcended the academic world and has become a cognitive and emotional impairment. What students feel, perceive, believe, and how they act directly influences this. In addition, what teachers feel and perceive, their expectations, beliefs and attitudes towards the discipline also play an important role in how they teach and in the affective dimension of their students. Based on theoretical aspects from various authors, this paper is aimed at addressing some elements regarding the affective dimension, and at showing elements pertaining to teachers and students, and their relationship with math learning and teaching. It was concluded that the role of the affective dimension in math learning must be addressed by math educators in order to understand the process from the perspective of the actors associated with it, both students and teachers, as well as to achieve a change in the discipline by improving the beliefs and attitudes of students and teachers.

Keywords. Attitudes, beliefs, students, teachers, teaching, learning, math.

Máster en Matemática Educativa por el Centro de Investigación y Estudios Avanzados del Instituto Politécnico Nacional, México. Licenciado en la Enseñanza de la Matemática por la Universidad Nacional. Se ha desempeñado como director, investigador y académico de la Escuela de Matemática de la Universidad Nacional. 
doi: http://dx.doi.org/10.15359/ree.18-2.6

URL: http://www.una.ac.cr/educare

CORREO: educare@una.cr

Las matemáticas se han convertido, dentro del currículo escolar, en una de las materias más temidas (que provocan, entre otros, un sentimiento de rechazo) y es una de las disciplinas en donde más bajos rendimientos se presentan. Lo anterior ha propiciado la construcción de una "imagen social negativa" en torno a ella y transmitida de una generación a otra.

A pesar de lo anterior, Hernández (2011) indica que habitualmente a las matemáticas se les ha relacionado con la racionalidad, la abstracción y el razonamiento lógico. En este sentido, Candia (2009) resalta que el aprendizaje de la disciplina debe estar vinculado con la formación de actitudes positivas hacia el conocimiento, pues estas constituyen un factor movilizador, al interior de los estudiantes, para construir la manera en que abordan la ciencia y sus disposiciones generadoras para potenciales transformaciones de los saberes compartidos en las experiencias educativas entre los protagonistas del proceso.

Pero estos protagonistas del proceso educativo ejercen su propia influencia en la enseñanza y aprendizaje de las matemáticas. Al respecto, Gómez (2000) indica que los profesores, estudiantes y padres de familia tienen su perspectiva sobre la disciplina, su enseñanza y aprendizaje, las cuales afectan las creencias del aprendiz, por lo que la toma de conciencia de la actividad emocional es un mecanismo de control personal en sus relaciones con las otras personas y en su autorregulación del aprendizaje. Se destaca, entonces, la importancia de la dimensión afectiva en el aprendizaje de las matemáticas y su influencia en este proceso.

Sobre ello, Díaz y Hernández (2010) señalan que entre los principios más valiosos que proporcionan un marco de referencia para las reformas curriculares y el rediseño de los procesos educativos están los factores motivacionales y afectivos del estudiantado. Dentro de estos, los autores destacan tres principios:

a) Las influencias motivacionales y emocionales sobre el aprendizaje, donde la motivación del estudiante determina qué y cuánto aprende, lo que está influido por estados emocionales, creencias, intereses, metas y hábitos de pensamiento de la persona que aprende.

b) Motivación intrínseca por aprender, la cual puede estimularse con tareas con un óptimo nivel de novedad y dificultad, relevantes desde los intereses del educando.

c) Efectos de la motivación sobre el esfuerzo, donde la adquisición de conocimiento y habilidades requiere de un trabajo intensivo por parte del estudiante y de la práctica guiada. Si no hay motivación en el estudiante, se reducen las posibilidades de que surja el deseo de realizar un esfuerzo por aprender.

Por lo tanto, el papel de la dimensión afectiva en el aprendizaje de las matemáticas debe ser un elemento abordado por la educación matemática como un medio para comprender este proceso desde la perspectiva de los actores relacionados con él, estudiantes y profesores, así como para lograr un cambio en dicha disciplina a partir del mejoramiento de las creencias y actitudes del alumnado y personal docente hacia ella. 
doi: http://dx.doi.org/10.15359/ree.18-2.6

URL: http://www.una.ac.cr/educare

CORREO: educare@una.cr

El presente artículo tiene como propósito abordar, basado en elementos teóricos de varios autores, algunos aspectos de la dimensión afectiva y, a partir de ellos, mostrar elementos relacionados con esta, tanto en docentes como estudiantes, y su relación con la enseñanza y aprendizaje de la disciplina.

Se pretende, tal como se muestra en la figura 1, describir la relación que la dimensión afectiva -compuesta por las emociones y sentimientos, creencias y actitudes- de estudiantes y profesores, posee en el aprendizaje de la disciplina.

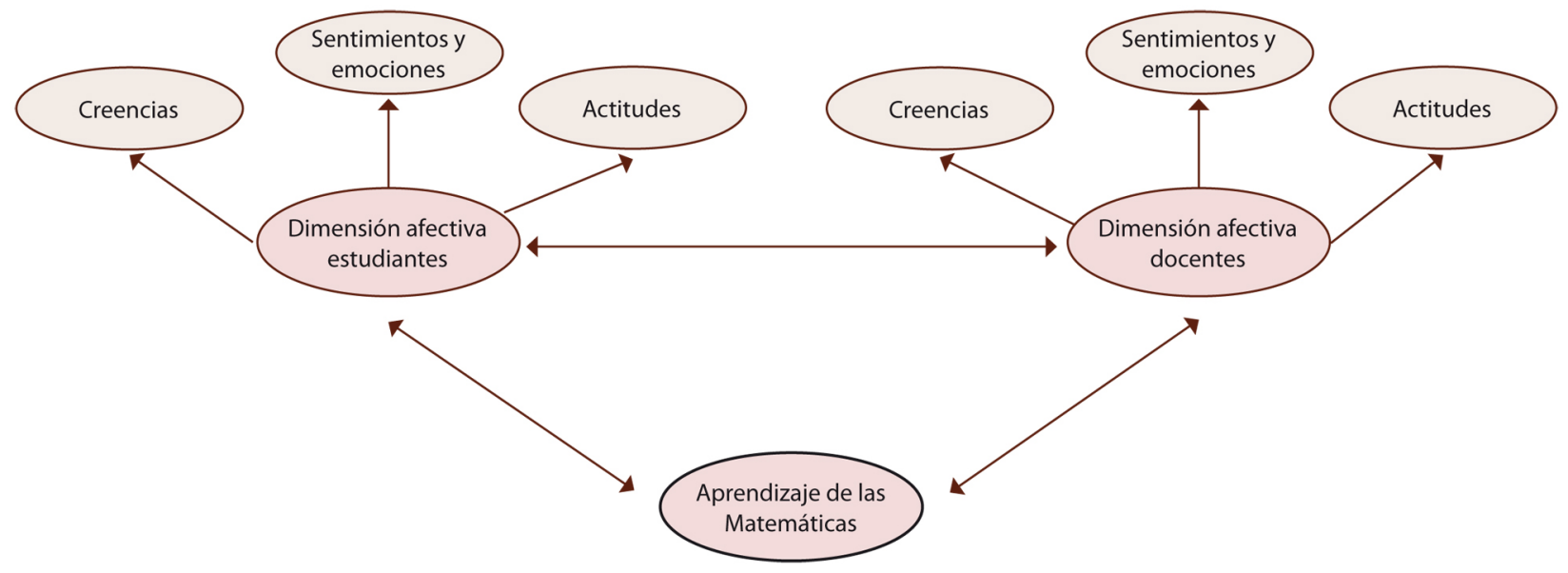

Figura 1. Relación de la dimensión afectiva y el aprendizaje de las matemáticas.

\section{Elementos de la dimensión afectiva}

Respecto a la dimensión afectiva, Gómez (2000) apunta que esta incluye actitudes, creencias, apreciaciones, gustos, preferencias, emociones, sentimientos y valores. La autora, basada en algunas investigaciones consultadas, define el término dimensión afectiva como "un extenso rango de sentimientos y humores (estados de ánimo) que son generalmente considerados como algo diferente de la pura cognición"(Gómez, 2000, p. 22), donde se considera no solamente los sentimientos y las emociones, sino también las creencias, las actitudes, los valores y las apreciaciones.

La relación entre la dimensión afectiva y aprendizaje no va en un único sentido, debido a que los efectos condicionan el comportamiento y la capacidad de aprender y recíprocamente el proceso de aprendizaje provoca reacciones afectivas (Estrada, 2002). Es decir, es un proceso cíclico.

En este sentido, Chaves, Castillo y Gamboa (2008) presentan la figura 2, modificado de Estrada (2002), con el propósito de ejemplificar las relaciones que se establecen entre los descriptores básicos de la dimensión afectiva en matemáticas. 
doi: http://dx.doi.org/10.15359/ree.18-2.6

URL: http://www.una.ac.cr/educare

CORREO: educare@una.cr

Al respecto los autores indican que:

[El esquema] evidencia la interacción cíclica con respecto al rol de los componentes del dominio afectivo en el proceso de enseñanza y aprendizaje de las Matemáticas. Al iniciar dicho proceso, los estudiantes tienen ciertas creencias sobre la disciplina y sobre sí mismo con respecto a su potencial para enfrentar su aprendizaje. En el proceso educativo recibe diversos estímulos que le generan cierta tensión, ante las que tiende a reaccionar emocionalmente, ya sea de forma positiva o negativa; pero, este comportamiento está condicionado por sus creencias previas. Las reacciones producidas generan sentimientos (emociones) de satisfacción, frustración, etc. Si las condiciones generadas en el aula se reiteran, las emociones se van solidificando hasta convertirse en actitudes positivas o negativas hacia las Matemáticas, su aprendizaje y hacia sí mismo; las cuales influyen en sus creencias originales y colaboran en su formación personal (Chaves et al., 2008, pp. 34-35).

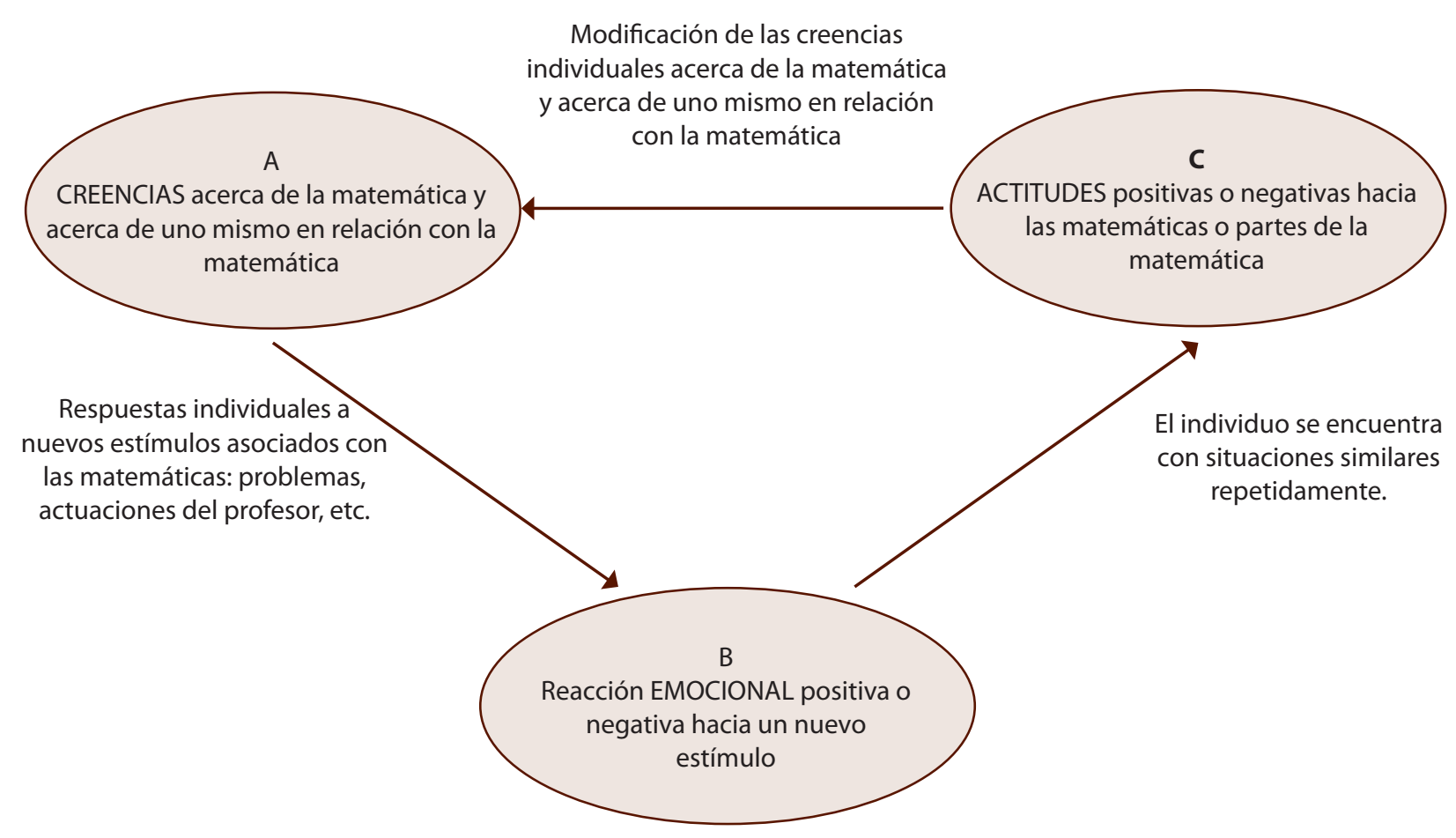

Figura 2: Dimensión afectiva en matemáticas y descriptores básicos. Nota:Tomado de Chaves, Castillo y Gamboa (2008). 
doi: http://dx.doi.org/10.15359/ree.18-2.6

URL: http://www.una.ac.cr/educare

CORREO: educare@una.cr

Si un estudiante asume el aprendizaje de la disciplina con la creencia de que es difícil y se enfrenta con una enseñanza que no le permite comprender los contenidos o resolver los ejercicios que se proponen en la clase, presentará frustración y hastío hacia la materia, se indispondrá con todo lo relacionado a ella y propiciará en él pensamientos negativos respecto a sí mismo en la materia, lo que podría convertirse en una creencia negativa de sí como aprendiz.

En este sentido, De Faria (2008) señala, respecto a las emociones, actitudes y creencias, que estas representan fuerzas impulsoras o de resistencia de la actividad matemática, por lo que si se desea mejorar la enseñanza y el aprendizaje de esta disciplina es conveniente tener en cuenta estos aspectos. Los trabajos realizados por McLeod (1988, 1989, 1992, 1994, citados por D. McLeod y S. McLeod, 2002), han dado una importante contribución para reconocer la trascendencia de estos aspectos, pues sus hallazgos permitieron determinar y explicar los efectos diferenciales del componente afectivo en dicho proceso. Este autor considera tres descriptores específicos: creencias, emociones y actitudes.

\section{Creencias}

Una creencia "es el conjunto de puntos de vista, de representaciones subjetivas que la persona va interiorizando (individualizando) y reforzando o debilitando en el decursar de su vida. Este sistema establece el contexto dentro del cual los recursos, la heurística y el control funcionan" (Sánchez, 2008, p. 3).

En el caso de las matemáticas, Parra (2005) señala que una creencia se entiende como el conjunto de conocimientos, valores e ideologías que posee un estudiante acerca de la disciplina. En el caso de sus docentes, el autor añade a lo anterior la manera como entiende todo lo referente a su enseñanza. Desde su perspectiva, estas creencias repercuten en todo el hacer profesional.

Adicionalmente, Mewborn y Cross (2007) indican que las creencias son personales, estables y frecuentemente están a un nivel superior del control inmediato; en general, son muy fuertes, ejercen una influencia sobre las acciones del individuo y son altamente resistentes al cambio; juegan un importante papel en las percepciones y conducta humana, por lo que pueden ser tomadas con distintos grados de convicción y no son consensuadas (Lazim, Abu y Wan, 2004; Thompson, 1992). Es decir, no se puede hablar de una "creencia general".

Pero tampoco podemos hablar de una creencia como un elemento aislado. Ponce, Martínez y Zuriaga (2008) señalan que una creencia nunca se sostiene con independencia de otras (sistemas de creencias) y que no se trata de una suma o yuxtaposición de estas, sino de una red organizada. En este sentido, los sistemas de creencias incluyen, a menudo, sentimientos afectivos y evaluaciones, vívidas memorias de experiencias personales (Cadoche y Pastorelli, 2005). 
doi: http://dx.doi.org/10.15359/ree.18-2.6

URL: http://www.una.ac.cr/educare

CORREO: educare@una.cr

En matemáticas las creencias de los individuos con respecto a esta están basadas en su experiencia en la disciplina, su enseñanza y aprendizaje, por lo que apoyado en la perspectiva matemática que expresa el alumnado y las creencias que este transmite, se puede realizar un diagnóstico de las experiencias que ha tenido y el tipo de enseñanza recibida en su aprendizaje (Gil, Blanco y Guerrero, 2005; Gómez, 2000).

En los estudios de McLeod (1989, 1992, citados por D. McLeod y S. McLeod, 2002), se logró determinar que las creencias individuales de los estudiantes sobre las matemáticas y su enseñanza, sobre sí mismo en su rol de aprendiz o sobre el contexto social tienen un fuerte impacto en el proceso educativo. Se mencionan dos categorías:

1) Creencias sobre las matemáticas como disciplina.

2) Creencias sobre sí mismo y su relación con las matemáticas.

Estrada (2002) indica que la primera de estas categorías considera la naturaleza de las matemáticas, donde los aspectos eminentemente afectivos son menos importantes. En Gil et al. (2005) se plantea que los alumnos creen que esta disciplina es útil, difícil y está fundamentada en reglas, lo que provoca diversas reacciones. Con esta creencia, la percepción de la utilidad de la disciplina se relaciona positivamente con el rendimiento. Según estos investigadores, las creencias surgen, generalmente, en el contexto escolar, particularmente en el aula.

Al respecto, Ernest (1988) señala que, en cuanto a la naturaleza de las matemáticas, existen tres visiones:

a) Instrumentalista: considera que las matemáticas constituyen una acumulación de hechos, reglas y habilidades que pueden ser usadas en la ejecución de algún fin externo.

b) Platónica: concibe que las matemáticas son un cuerpo de conocimientos estático y unificado; son descubiertas, no creadas.

c) Resolución de problemas: visualiza las matemáticas como un campo de creación e invención humana en continua expansión, que son un producto cultural no acabado y sus resultados están abiertos a la revisión.

Por ello se señala que "según la visión particular acerca de las matemáticas, se puede propiciar en los estudiantes diferentes tipos de aprendizaje que pueden ser memorísticos y algorítmicos o, por el contrario, aprendizaje que requieran del alumno un pensamiento creativo para enunciar conjeturas, aplicar de manera razonada la información, descubrir y, en general, construir su conocimiento" (Mora y Barrantes, 2008, p. 72).

Para muchas personas esta disciplina se caracteriza por resultados precisos y procesos infalibles, cuyos elementos básicos son las operaciones aritméticas, procesos algebraicos, términos geométricos y teoremas (Thompson, 1992). Desde esta perspectiva, señala la autora, saber matemáticas es equivalente a ser exitoso en el manejo de procedimientos y ser capaz de identificar los conceptos básicos de la disciplina. 
doi: http://dx.doi.org/10.15359/ree.18-2.6

URL: http://www.una.ac.cr/educare

CORREO: educare@una.cr

La segunda categoría, Estrada (2002) la relaciona con aspectos afines al aprendizaje. Sobre ellos los estudiantes poseen diversas expectativas, tales como la forma en que el aprendizaje debe llevarse a cabo, el papel que debe jugar el profesor, la metodología empleada, así como el rol que tiene el contexto al que pertenecen. Dicha categoría incorpora elementos relacionados con el autoconcepto, la confianza, entre otras, donde el primero se convierte en un buen predictor del rendimiento académico en la disciplina (Gil et al., 2005).

Se debe tener claro que no nacemos con un conjunto de creencias, sino que son el resultado de un proceso evolutivo en el que el autoconcepto, la confianza en sí mismo y la autoeficacia percibida juegan un papel fundamental (Ponce et al., 2008). En este sentido, Liljedahl (2005) indica que la creencia personal de un individuo sobre su habilidad para hacer matemáticas está relacionada con su autoeficacia, la cual es producto de sus experiencias con la disciplina.

Por ello, el autoconcepto en relación con la matemáticas está constituido por conocimientos subjetivos (creencias y cogniciones) y las emociones e intenciones relativas a ella; está relacionado con el interés hacia esta y los intereses (motivos, finalidades) respecto a la materia, las razones asociadas a la motivación y el placer al enfrentarse a la disciplina, la eficiencia, atribución causal del éxito o fracaso escolar y el autoconcepto como miembro de un determinado grupo social (Gómez, 2000).

\section{Sentimientos y emociones}

Los sentimientos "son nuestras respuestas desarrolladas cultural y ambientalmente a las circunstancias" (Jensen, 2010, p. 105) e incluyen la preocupación, anticipación, frustración, cinismo y optimismo, entre otros.

Relacionado con esto, Chaves et al. (2008) señalan que se puede decir"que los sentimientos constituyen un factor clave al momento de comprender o tratar de explicar el desenvolvimiento de un estudiante en las lecciones de Matemáticas" (p. 33). Por ejemplo, el miedo al fracaso en dicha disciplina es una construcción afectiva complicada basada en varios factores como el contexto familiar, las características de los estudiantes y la práctica de los profesores, los cuales deben ser considerados y abordados para comprender las distintas situaciones que ocurren en el aula de matemáticas (Pantziara y Philippou, 2011).

Respecto a las emociones, se puede concretar que ellas se corresponden con un fenómeno de tipo afectivo que un sujeto emite en respuesta a un suceso, interno o externo, que tiene para él una carga de significado. Estas reacciones psico-físicas, de carácter momentáneo, suelen estar acompañadas de expresiones orgánicas, características asociadas con pensamientos, motivaciones, experiencias, elementos hereditarios, cogniciones, estados psicológicos y biológicos y tendencias de actuar. (Martínez, 2008, p. 249) 
doi: http://dx.doi.org/10.15359/ree.18-2.6

URL: http://www.una.ac.cr/educare

CORREO: educare@una.cr

Por su parte, Gil et al. (2005) apuntan que "Las emociones son respuestas organizadas más allá de la frontera de los sistemas psicológicos, incluyendo lo fisiológico, cognitivo, motivacional y el sistema experiencial. Surgen en respuesta a un suceso, interno o externo, que tiene una carga de significado positiva o negativa para el individuo" (Gil et al., 2005, p. 23). Estas se generan por vías biológicamente automatizadas e incluyen el gozo (placer), el miedo, la sorpresa, el disgusto, la ira y tristeza; son parte de la personalidad y ayudan a tomar decisiones (Jensen, 2010).

Las emociones desencadenan los cambios químicos que alteran nuestros estados de ánimo, conductas y, finalmente, nuestras vidas. Si la gente y las actividades son el contenido de nuestras vidas, las emociones son tanto los contextos como los valores que tenemos. Simplemente no podemos dirigir un centro docente sin reconocer las emociones e integrarlas en las actividades diarias. (Jensen, 2010, p. 110)

Por lo tanto, las emociones son intrínsecas a la persona y son un reflejo de sus sentimientos, por lo que no se pueden considerar en forma aislada. En este sentido, Gómez (2000) indica que una reacción emocional es una respuesta afectiva fuerte, "visceral", de corta duración y que es el resultado de discrepancias entre lo que el sujeto espera y lo que se produce en el momento en que la reacción se experimenta, por lo que las emociones son respuestas afectivas fuertes, no automáticas y son el resultado complejo del aprendizaje, de la influencia social y de interpretación.

En el modelo cognitivo, los sentimientos y emociones, tales como alegría, miedo, enojo, ansiedad, tristeza, entre otros, no dependen de una determinada situación, sino de cómo la interpreta la persona (Ponce et al., 2008). En la enseñanza de las matemáticas, las actividades didácticas que el docente plantea no son percibidas de la misma forma por todos sus estudiantes y la emociones que estas generen en cada uno de ellos dependerán de lo que sientan, cómo perciban al profesor, la asignatura y cómo se enfrenten a esta. Algunos expresan satisfacción, reto, alegría y ánimo; mientras que otros sienten desesperación, tristeza, desánimo y estrés, lo que puede contribuir o afectar su aprendizaje.

En el caso de las emociones, Candia (2009) plantea que si se pueden cambiar las evaluaciones, interpretaciones y atribuciones que se hacen de los hechos reales, se pueden cambiar las emociones de miedo y la ansiedad que se puede experimentar al "enfrentarse" a algunos de ellos, pues cuando una persona está ansiosa interpreta los sucesos como amenazantes y peligrosos.

Conocer las emociones que generan en estudiantes las matemáticas, sus docentes, las actividades que se proponen en el aula y las causas de estas, sirven de base al profesorado para generar propuestas de cambio que se orienten a modificar las emociones negativas y potenciar las positivas en procura de un aprendizaje significativo. 


\section{Actitudes}

Las actitudes "son experiencias subjetivas (cognitivo-afectivas) que implican juicios evaluativos, que se expresan en forma verbal o no verbal, que son relativamente estables y que se aprenden en el contexto social" (Díaz y Hernández, 2010, p. 45).

La actitud se define como la predisposición evaluativa (es decir, positiva o negativa) que determina las intenciones personales e influye en el comportamiento. Consta, por lo tanto, de tres componentes: una cognitiva, que se manifiesta en las creencias subyacentes a dicha actitud; una componente afectiva, que se manifiesta en los sentimientos de aceptación o de rechazo de la tarea o de la materia; y una componente intencional o de tendencia hacia un cierto tipo de comportamiento. (Gil et al., 2005, p. 20)

Por lo tanto, la formación de actitudes está relacionada con la experiencia personal y social que cada individuo vive. En este sentido, "una actitud puede definirse como una organización aprendida y relativamente duradera de creencias acerca de un objeto o de una situación, que predispone a un individuo en favor de una respuesta preferida" (Bernal, 2009, p. 18).

La actividad matemática está sumergida en un contexto cultural y se ve afectada por la interacción de diferentes actores del proceso educativo (estudiantes, docentes, directivos, padres de familia, entre otros) donde cada uno de ellos tiene una actitud diferente hacia la disciplina e influye socialmente sobre los otros, afectando los procesos interpersonales (sentimientos, motivación, procesamiento de la información) y sobre las valoraciones hacia las matemáticas (Bernal, 2009). Según el autor, este es un factor que afecta el proceso enseñanza- aprendizaje de la materia.

Uno de los contenidos poco atendidos en la enseñanza en todos los niveles y materias es el de las actitudes, a pesar de que es ampliamente reconocida su importancia y que se ha demostrado que muchas de las actitudes se desarrollan en el seno de las instituciones escolares, por medio de un aprendizaje incidental y un currículo oculto donde el profesor es quien, de forma directa o indirecta, se enfrenta a esta problemática (Díaz y Hernández, 2010).

El aprendizaje de las actitudes es un proceso lento y gradual que se ve influido por las experiencias personales previas, las actitudes de otras personas significativas, la información y experiencias relevantes y el contexto sociocultural (Díaz y Hernández, 2010). Esta situación, sin embargo, sobrepasa la institución escolar, pues inciden en ella el contexto familiar, los medios de comunicación y la sociedad, los cuales promueven, en muchos casos, valores y actitudes que atentan lo que se espera lograr en la escuela.

En este sentido, Hernández (2011) apunta que las actitudes que se exteriorizan se relacionan tanto con el objeto como con el contexto o situación en la que se desarrolla el sujeto. Por esto, indica el autor, el comportamiento es el resultado de la mezcla de las actitudes inducidas por el objeto y de las actitudes propiciadas por la situación. 
doi: http://dx.doi.org/10.15359/ree.18-2.6

URL: http://www.una.ac.cr/educare

CORREO: educare@una.cr

En el caso de las matemáticas, Akay y Boz (2010) indican que existe una gran cantidad de estudios sobre la actitud hacia estas, llevados a cabo en diversas áreas considerando aspectos como etnia, inteligencia, métodos de enseñanza y contextos socioeconómicos, los cuales han concluido que la motivación, la actitud y la propia eficacia podrían ser buenos predictores para el aprendizaje de la disciplina.

Al respecto, Martínez (2008) expresa que las actitudes hacia las matemáticas tienen que ver con la valoración, satisfacción, curiosidad, el aprecio e interés por la disciplina y su aprendizaje, acentuando más el componente afectivo que el cognitivo, y se caracterizan por considerar las capacidades de los sujetos y su modo de utilizarlas. Por ejemplo, el autor menciona que se pueden observar situaciones donde las matemáticas son valoradas y apreciadas por la posibilidad que poseen para resolver problemas cotidianos y de aplicarla en otras ramas del conocimiento; o también por su belleza, potencia y simplicidad al ser usada como lenguaje y estar conformadas por métodos propios.

Sobre este aspecto, Pezzia y Di Martino (2011) caracterizan la actitud hacia las matemáticas y su enseñanza en tres dimensiones relacionadas entre sí: la visión de la disciplina y su enseñanza, la competencia percibida en la enseñanza de esta y la disposición emocional hacia la materia y su enseñanza.

En este sentido, Belbase (2010) manifiesta que hay ocho posibles resultados del modelo de representación de las diferentes percepciones acerca de las matemáticas:

1. infalibles, alta autoestima, actitud positiva;

2. infalibles, alta autoestima, actitud negativa:

3. infalibles, baja autoestima, actitud positiva;

4. infalibles, baja autoestima, actitud negativa;

5. falibles, alta autoestima, actitud positiva;

6. falibles, alta autoestima, actitud negativa;

7. falibles, baja autoestima, actitud positiva;

8. falibles, baja autoestima, actitud negativa.

El autor menciona que entre estas combinaciones, la (1), (4), (5) y (8) son situaciones prácticamente viables, pues aunque las restantes son teóricamente viables parecen no ser prácticas ya que una alta autoestima y una actitud negativa y una baja autoestima y actitud positiva hacia las matemáticas parecen contradecirse. La contradicción en la actitud de una autoestima alta y actitud negativa, y la baja autoestima y una actitud positiva es evidente, ya que representan caracteres opuestos acerca de la percepción hacia la disciplina.

Entre las cuatro posibilidades con la primera combinación de infalible (imagen), una autoestima alta y una actitud positiva, es posible desarrollar una percepción respecto a las 
matemáticas como absoluta, infalible e incorregible, aunque el estudiante tenga una alta autoestima y una actitud positiva hacia ellas. La visión de estas como algo absoluto e infalible lleva al estudiante a desarrollar una filosofía positivista, que puede conducir al desarrollo de su personalidad como un absolutista. El estudiante con este tipo de personalidad disfruta de la rutina de resolución de problemas, sigue un procedimiento rígido para enfrentarse a ellos y obtiene altos puntajes en las pruebas.

La combinación de la cuarta parte de infalible, baja autoestima y la actitud negativa es una situación problemática. Con base en el profesor, se podría decir la enseñanza dada a un estudiante que presenta dicha combinación se caracterizaría por presentar pocas actividades para este, escasa interrogación y poco énfasis en el trabajo en grupo, por lo que la instrucción autoritaria puede resultar en una baja autoestima y la actitud negativa hacia las matemáticas. La enseñanza y el aprendizaje de la materia, guiados por la "copia y la práctica", sin construcción de las ideas por parte los estudiantes, posiblemente, lleva a esta situación con un grave impacto en el rendimiento estudiantil.

La combinación de la quinta parte de falibles, alta autoestima y actitud positiva implica desarrollar una percepción de que los objetos matemáticos son construidos socialmente, falibles y cuestionables, y que el estudiante posee una alta autoestima respecto a la disciplina, lo que conlleva a una actitud positiva. Esta combinación desarrolla la personalidad de los estudiantes respecto a los objetos matemáticos, manteniendo una alta autoestima sobre el aprendizaje de la materia y pensar de manera positiva sobre su capacidad para aprender. Estos estudiantes valoran el proceso de aprendizaje y tratan de comprender la naturaleza de las matemáticas a partir de ejemplos y prácticas. Disfrutan de los problemas no rutinarios.

La octava combinación de falibles, baja autoestima y la actitud negativa conduce a desarrollar una percepción de que los objetos matemáticos son construidos socialmente, falibles y cuestionables; sin embargo, el estudiante tiene una baja autoestima debido a algunos problemas internos y externos para hacer frente a la situación en el aula que, en última instancia, conduce al desarrollo de actitudes negativas. El profesor puede ayudar a estos estudiantes a desarrollar una autoestima alta, cambiando el ritmo de aprendizaje y ayudándoles para aprender del contexto con la solución de problemas no estructurados.

\section{La dimensión afectiva de los docentes de matemáticas}

Bishop et al. (1993, citados por Belbase, 2010) identificaron cuatro grupos de influencias que parecen ser cruciales para los estudiantes respecto a matemáticas: la influencia de la sociedad, el contexto socio-cultural del pensamiento matemático, la influencia de los recursos para la enseñanza y el papel del maestro en el aprendizaje de la disciplina. En este sentido, las creencias personales del profesor y las actitudes desempeñan un papel importante en el abordaje de estos factores, pues la metodología que desarrolle en el aula da forma a imágenes de las matemáticas en la mente de los alumnos y contribuye en el desarrollo de sus actitudes hacia ellas. 
doi: http://dx.doi.org/10.15359/ree.18-2.6

URL: http://www.una.ac.cr/educare

CORREO: educare@una.cr

En este sentido, Lamas (2010) apunta, respeto al profesor, que sus características personales, método de enseñanza, estilo docente, actitud hacia la diversidad, experiencia profesional y competencia profesional ejercen influencia en el aprendizaje del alumnado. Un docente poco accesible, intimidante, que planifica el proceso de enseñanza centrado en él y su trabajo, que no considere las diferencias individuales y cuya preparación profesional no haya sido acorde al contexto educativo en el cual se desarrolla, generara una mala imagen en el estudiante y hacia la materia.

El constructivismo postula que la educación debe partir del conocimiento, actitudes e intereses del estudiantado y que estos construyen su conocimiento a partir de la interacción entre estos elementos, la experiencia y su entendimiento (Howe y Berv, 2002). Por ello, la función del educador es favorecer experiencias que les permitan dicha interacción.

Pero, además de lo anterior, el personal docente debe ser consciente del papel que su dimensión afectiva posee en la estructuración de este en sus estudiantes. "El profesor es siempre un importante agente socializador, un modelo de valores, comportamientos y actitudes, un otro significativo que ejerce su influencia y estatus de poder legitimados institucionalmente, para promover actitudes positivas (o negativas) en sus alumnos" (Díaz y Hernández, 2010, p. 46).

Al abordar el impacto de las creencias del profesorado sobre sus prácticas de enseñanza, se deben considerar la influencia del contexto social y el nivel de consistencia de las propias creencias (Gómez, 2000). La autora señala que el contexto social está configurado por las expectativas de estudiantes, profesores, padres y de otras instituciones, las cuales brindan oportunidades o restricciones a la situación de enseñanza.

Cuando quien ejerce la docencia llega a una clase trae una visión de sus estudiantes que influye en lo que les va a proponer, cómo lo hace y cómo lo va a valorar; de igual forma la percepción del alumnado sobre el profesor o profesora va a afectar la interpretación de dichas propuestas (Solé, (1999).

La autora señala que de acuerdo con lo que los docentes esperan de los estudiantes, estos proporcionan, a veces inconscientemente, tratamientos educativos diferenciados, que se traducen en el tipo y grado de ayuda educativa que se les brinda, apoyo emocional y retroalimentación positiva (o negativa) que reciben, los tipos de actividades en las que se les permite participar, oportunidades de aprendizaje ofrecidas, cantidad y dificultad de los recursos educativos aportados, entre otros, que pueden variar según cómo se considere al alumnado.

Parecer ser que los profesores creemos que nuestra influencia y control es mayor sobre los considerados buenos alumnos. Todavía en relación a éstos, tendemos atribuir sus éxitos a causas internas (como la capacidad) y sus fracasos a causas externas o situacionales (como la dificultad intrínseca de la tarea). Sin embargo, cuando el alumno no es tan bueno, atribuimos sus fracasos a causas internas (como su escasa capacidad) y los éxitos a causas externas, como la suerte, la simplicidad de la tarea o bien a causas internas no estables, como el esfuerzo: "aunque no es brillante, ha podido hacerlo, porque ha esforzado mucho". (Solé, 1999, p. 37) 
doi: http://dx.doi.org/10.15359/ree.18-2.6

URL: http://www.una.ac.cr/educare

CORREO: educare@una.cr

Al respecto, Solé (1999) plantea que si un profesor considera que el fracaso de un buen alumno se debe a que ese día estaba despistado o a que la tarea estaba complicada, tenderá a modificar aspectos de esta que la hagan manejable, lo que permite que la labor inabordable se convierta en un reto, posibilite el aprendizaje y favorezca el aprendizaje emocional. Pero, por el contrario, cuando el fracaso es atribuido a la poca capacidad del alumno, el docente actúa de forma distinta, pues se basa en la escasa confianza en el estudiante para aprovechar las ayudas brindadas, con lo que este pierde la oportunidad de aprender y de experimentar aprendizaje.

La acción del docente de matemáticas se debate entre las intenciones personales de lo que se considera su deber ser profesional y las expectativas sociales que las distintas personas relacionadas con el entorno tienen en relación con su hacer profesional (Parra, 2005). El personal docente podría consideraren la necesidad de formación matemática sólida y , y creer, estricta, y creer en ello; sin embargo, el entorno muchas veces difiere de esta idea e incentiva el poco esfuerzo y a estudiar para aprobar, lo que genera un choque para el docente.

Ernest (1988) expone que las reformas en la enseñanza de las matemáticas no pueden ocurrir sin que las creencias de los profesores, su enseñanza y aprendizaje cambien. Según el autor, estas están ligadas al menos a tres componentes: visión de la naturaleza de las matemáticas, visión de la naturaleza de su enseñanza y visión acerca de los procesos de aprendizaje de la disciplina.

Las creencias de docentes sobre la naturaleza de las matemáticas y el aprendizaje, la profundidad del conocimiento matemático y las percepciones sobre la habilidad matemática influencian sus creencias sobre lo que significa aprender y hacer matemáticas, y determina su enseñanza y prácticas pedagógicas (Benken, 2005). En este sentido, además, cuando se enseña, aprende o evalúa un aprendizaje matemático en un determinado contexto, intervienen complejas situaciones cargadas de significados que se dan entre estudiantes, sus docentes y el saber matemático (Ponce et al., 2008).

Si el profesorado considera que la disciplina se aprende por medio de reglas y procedimientos, esto será lo que incentive en sus lecciones. Por el contrario, si cree que el aprendizaje de esta es por medio de una construcción social, las tareas implementadas perseguirán ese objetivo. La importancia de que el docente sea consciente de sus creencias radica en que, si esto se logra, puede cuestionar las evidencias de ellas y, por ende, las relaciones entre estas y su práctica pedagógica (Mewborn y Cross, 2007).

En este sentido, Mewborn y Cross (2007) y Benken (2005) indican que las creencias de los docentes son una ventana para entender sus acciones, experiencias, cómo ellos interpretan los eventos y puede ayudar, además, a entender los procesos de enseñanza y aprendizaje de las matemáticas. La visión del docente sobre su rol como profesor de la materia y las percepciones sobre el afecto de sus estudiantes están relacionadas con el contexto y como cree él que aprenden los estudiantes. 
doi: http://dx.doi.org/10.15359/ree.18-2.6

URL: http://www.una.ac.cr/educare

CORREO: educare@una.cr

Dado que los aspectos cognitivos y emocionales pueden explicar el éxito o fracaso en matemáticas, Ponce et al. (2008) indican que los docentes deben ser transmisores no solo de herramientas cognitivas, sino de expectativas positivas y de motivaciones de logro.

Para lograr una mejora en las actitudes hacia la disciplina, es necesario realizar un cambio en la metodología que se utiliza en el aula para su enseñanza, que impacte positivamente en la imagen de la misma y considere a las personas a quienes va dirigida, a partir de sus características afectivas, cognitivas y contextuales (Ponce et al., 2008). El papel del personal docente en este proceso es fundamental.

\section{La dimensión afectiva de los estudiantes}

Distintos investigadores han puesto de manifiesto que los efectos de las emociones, actitudes y creencias de los estudiantes son factores claves en la comprensión de su comportamiento en matemáticas (Gil et al., 2005). Incluso, Hernández (2011) es enfático en señalar que la evidencia empírica presentada ha demostrado que las creencias de los estudiantes hacia la disciplina tienen influencia en la selección de una carrera universitaria.

Las emociones de los estudiantes por las matemáticas están relacionadas con frustración y hastío hacia la asignatura; aunado a lo anterior, la experiencia diaria indica que tampoco hay en la sociedad una buena percepción del profesorado de la materia, en general, y que no se les considera en un mismo nivel que otros profesionales (Chandía, Quiroga y Ulloa, 2006).

Lamas (2010, basado en Cueto, 2004) y Soares (2004) apuntan que los factores que determinan el rendimiento escolar de los estudiantes pueden ser clasificados en tres grandes categorías:

a) Factores familiares (características socioeconómicas y culturales)

b) Factores escolares (infraestructura escolar, prácticas didácticas, recursos educativos, características de los profesores, entre otros)

c) Factores relacionados al alumnado (habilidades, motivación, actitudes, entre otros)

En este último aspecto la dimensión afectiva de los estudiantes adquiere importancia. En el aprendizaje intervienen diversos aspectos de tipo afectivo y relacional donde el aprendizaje y el éxito influyen en la construcción del concepto que tenemos de nosotros, en la estima que nos profesamos y en las capacidades relacionadas con el equilibro personal (Solé, 1999).

Por esta razón, Gil, Guerrero y Blanco (2006) recalcan la importancia de comprender y analizar cómo los estudiantes, al aprender matemáticas e interactuar en su entorno, adquieren determinadas creencias y valoraciones positivas o negativas hacia ellas y sobre sí mismo respecto a estas, lo que le genera éxitos o fracasos en la materia. 
Al respecto, Ponce et al. (2008) señalan que investigar la imagen que los estudiantes tienen de las matemáticas y de los sentimientos que estas les producen pueden servir de referencia para realizar un análisis crítico de los métodos y estrategias utilizadas en la enseñanza de la disciplina y tener visión de la perspectiva matemática del alumnado, sus creencias y pensamientos respecto a su uso, a cómo debe ser enseñada, a cómo se aprende, y tener una aproximación de las experiencias en la materia.

Sánchez (2008) indica que las creencias de los estudiantes en las matemáticas están relacionada con cuatro ejes

a) Sobre sí mismo.

b) Sobre el entorno (papel que cree jugar o desempeñar ante su profesor, compañeros, entre otros).

c) Sobre el problema (si es capaz de resolverlo y la reglas para hacerlo).

d) Sobre las Matemáticas.

Sobre el docente, Solé (1999) indica que las representaciones que los estudiantes construyen sobre sus profesores están relacionadas con factores afectivos, la disponibilidad que muestra, el respeto y afecto que transmite, la capacidad de mostrarse acogedor y positivo. La autora señala que en la educación secundaria a estos factores se les debe sumar el conocimiento por la materia, capacidad para motivar e implicar a los estudiantes, claridad para realizar sus explicaciones, temas tratados, entre otros.

Las imágenes de las matemáticas están determinadas, en gran parte, por las perspectivas epistemológicas y filosóficas con que cada persona ve la disciplina, ya sea a priori o a posteriori, las cuales, según la percepción individual, tienen un papel importante en el desarrollo de actitudes hacia ella en el largo plazo (Belbase, 2010). En este sentido, señala el autor, las imágenes metafóricas que poseen los estudiantes sobre la asignatura desempeñan un papel importante en el desarrollo de las creencias y actitudes hacia esta disciplina, en términos de tener una opinión favorable o desfavorable.

Si el alumno, ya sea por el contexto familiar o por sus compañeros, tiene la imagen de las matemáticas como una materia difícil y se enfrenta con actividades que requieren habilidades o destrezas que no ha desarrollado y con un personal docente que no facilita la comprensión de los contenidos, esta idea se reforzará y afianzará para toda la vida. Pero, por el contrario, si las tareas son accesibles a él, le permiten demostrar los conocimientos adquiridos y el profesorado se constituye en un guía del proceso, esta la imagen original podría sufrir modificación e influenciar, a la vez, en su motivación para el estudio de la materia.

Los estudiantes continuamente reciben información sobre qué significa aprender matemáticas y el significado social de aprenderlas, por lo que su autoconcepto como aprendiz de la disciplina está relacionado con sus actitudes, perspectiva del mundo "matemático" y su identidad social (Gómez, 2000). 
doi: http://dx.doi.org/10.15359/ree.18-2.6

URL: http://www.una.ac.cr/educare

CORREO: educare@una.cr

Las creencias que los estudiantes poseen sobre las matemáticas tienden a convertirse en "realidades" respecto a la disciplina y están frecuentemente asociadas a experiencias que han vivido en el estudio de esta materia (Liljedahl, 2005). Este autor señala que de estas experiencias han surgido ideas como la dificultad de la disciplina, su utilidad, que su estudio gira en torno a la obtención de una respuesta y que se basa en memorizar fórmulas, entre otras, que luego ellos fueron reforzando a lo largo de su desarrollo académico.

Sin embargo, a pesar de su utilidad, esta disciplina suele ser percibida por los estudiantes como una materia difícil, aburrida, poco práctica, abstracta, entre otros, cuyo aprendizaje requiere de una "habilidad especial" y que no está siempre al alcance de todos (Gil et al., 2006). Por ello, señalan los autores, esta disciplina se ha convertido en una fuente de frustración, desánimo y angustia.

El proceso de enseñanza y aprendizaje de las matemáticas y las influencias de naturaleza cognitiva y emocional se presentan entrelazadas, pues las dificultades que el estudiantado enfrenta en el logro académico en la disciplina están relacionadas con el grado de conexión entre el método matemático y su autoconcepto (Hernández, 2011). Desde esta perspectiva, afirma el autor, señalar que las matemáticas son abstractas o aburridas presenta una idea preconcebida que el individuo tiene hacia la disciplina.

Sobre la influencia de los estados emocionales en el aprendizaje, Jensen (2010) señala que las experiencias vividas generan emociones como temor, miedo, sorpresa, entre otras; que originan pensamientos, opiniones y decisiones; que producen respuestas como cinismo, optimismo, confianza, frustración o confusión, y que influyen en la motivación del discente para ejecutar una acción.

Estudiantes con dificultades en el aprendizaje de la materia, frecuentemente, al dudar de sus capacidades, exageran la magnitud de sus deficiencias y tienden a atribuir su fracaso a su falta de capacidad. Por el contrario, cuando poseen éxito este es atribuido a la dificultad del problema (fácil), a la ayuda del docente, compañeros o a la suerte (Gil et al., 2006). Apunta Solé (1999) que, incluso, puede ocurrir que la ansiedad -que en cierto grado está presente en todo proceso de aprendizaje- se vuelva demasiado intensa, provocando miedo al fracaso y la adopción de enfoques de aprendizaje superficiales que lo eviten, principalmente orientados a cumplir con los requisitos solicitados.

El predominio de factores afectivos y emocionales en el aprendizaje de la disciplina se observa, en el estudiante, en la ansiedad que muestra ante el planteamiento de problemas que requieren de una resolución compleja, la sensación de malestar, frustración, inseguridad y bajo autoconcepto, lo que les impide enfrentar con eficacia sus tareas matemáticas y les lleva a abandonar las actividades, reforzando la creencia de ser incapaces de alcanzar el logro académico en la disciplina (Hernández, 2011).

Por ello, la formación estudiantil en el ámbito emocional y afectivo se debe fundamentar en la importancia que poseen los pensamientos y creencias en la explicación del comportamiento ante las actividades propuestas, los cuales explican los rechazos y el gusto hacia la disciplina, el profesorado y la situación de aprendizaje (Hernández, 2011). 
doi: http://dx.doi.org/10.15359/ree.18-2.6

URL: http://www.una.ac.cr/educare

CORREO: educare@una.cr

Cuando las emociones se suprimen o expresan de forma inadecuada, se presentan problemas de disciplina, por lo que una función de los profesores es activar emociones productivas; desde esta perspectiva, se debe tener presente que una buena enseñanza no es aquella que evita las emociones, sino las que las abarca, las contempla en sus actividades y la que examina lo que los alumnos necesitan aprender para desarrollar las destrezas de la inteligencia emocional de un modo repetitivo, con el propósito de que las conductas positivas sean tan "automáticas" como las negativas (Jensen, 2010).

En este sentido, la relación que se establece entre los efectos y el aprendizaje es cíclica:

De una parte, la experiencia que tiene el estudiante al aprender matemáticas le provoca distintas reacciones emocionales e influye en la formación de creencias; por otra, las creencias que sostiene el sujeto tienen una consecuencia directa en su comportamiento en situaciones de aprendizaje y en su capacidad para aprender. (Gil et al., 2005, p. 17)

Por esta razón, considerar "cuál es el estudiante o la estudiante" que se tiene en el aula, sus sentimientos, creencias y actitudes, y la forma en que perciben las matemáticas y su aprendizaje debe ser una prioridad en todo contexto educativo como un mecanismo para lograr un aprendizaje significativo que no solo responda a la necesidades intelectuales de sino también afectivas y emocionales.

\section{Reflexiones finales}

Las matemáticas se han convertido, para un número importante de estudiantes, en un obstáculo para el logro de sus objetivos escolares. Pero, lamentablemente, para muchos de ellos esta dificultad ha trascendido la parte académica y se ha establecido como un impedimento cognitivo y emocional condicionado por lo que siente y percibe, por sus expectativas, creencias y actitudes respecto a la disciplina.

Aunado a lo anterior, lo que el personal docente de matemáticas siente y percibe, sus expectativas, creencias y actitudes respecto a la disciplina también juegan un importante papel en el tipo de enseñanza que realiza y la dimensión afectiva de sus estudiantes.

La importancia de abordar la dimensión afectiva en los procesos de enseñanza y aprendizaje de la asignatura es un hecho que no se puede obviar. En ese sentido, Lamas (2010) señala que una educación matemática de calidad será aquella que proporcione a los estudiantes herramientas para actuar en una variedad de situaciones de la vida diaria que implican no solo conocimiento matemático, sino estrategias afectivas y emocionales para desenvolverse correctamente y enfrentar dichas situaciones.

Alrededor de las matemáticas se ha creado una atmósfera negativa donde la sociedad y el contexto ejercen una gran influencia, la cual también afecta en forma directa al estudiante. Sin embargo, tal y como lo señala Hernández (2011), el individuo, como ser social, no está 
doi: http://dx.doi.org/10.15359/ree.18-2.6

URL: http://www.una.ac.cr/educare

CORREO: educare@una.cr

determinado por el temperamento y su modo de ser en la conducta emotiva puede ser modificado por la educación.

Desde esta perspectiva, se puede decir que es una obligación de todos los involucrados en la educación matemática abordar el tema de la dimensión afectiva y trabajar en propuestas tendientes a la modificación de aquellas que no permiten el logro de aprendizajes significativos. Por esta razón, se hace necesario realizar cambios en el sistema educativo orientados a tomar en cuenta el contexto del estudiante, sus creencias, actitudes y conocimientos previos, sus capacidades y particularidades.

En este sentido, en esta reflexión se coincide con Solé y Coll (1999) al señalar que la escuela debe favorecer el bienestar y el desarrollo de su alumnado en las dimensiones social, equilibrio personal y cognitivas, donde se brinde un clima favorable para el aprendizaje, el profesorado trabaje en equipo, exista una dirección eficaz y estabilidad en la condición docente, el currículo se planifique cuidadosamente, los padres apoyen la tarea educativa, existan unos valores propios de la escuela y se cuente con el apoyo activo de las autoridades educativas responsables (cuya misión debe se debe centrada en facilitar los cambios necesarios).

Autores como Akay y Boz (2010) señalan que es muy importante mejorar las creencias y actitudes de los estudiantes hacia las matemáticas, así como las creencias respecto a su eficacia en la disciplina. El cuerpo docente debe tener claro que, cuando se desea aprender y se aprende, dicha experiencia le proporciona al individuo una imagen positiva de sí mismo y refuerza su autoestima (Solé, 1999). Cómo lograr este deseo por aprender debe ser un reto que el profesorado debe convertir en un ideal y en una meta a seguir en su labor diaria.

La enseñanza de las matemáticas como un "modelo" donde el profesor "es el que sabe", el que dice qué y cómo hay que resolver los problemas, el que es concebido casi con un ser humano "místico y con capacidades especiales" que no necesita relacionarse con sus alumnos ni conocerlos más allá de su papel en el aula, y donde el estudiante es un receptor pasivo, cuya "inteligencia" se "mide" por un número, ha dejado de ser efectiva. Son los mismos cuerpos docentes que reclaman un cambio.

Esta disciplina no debe ser vista como un obstáculo, un trauma, un impedimento o un sufrimiento. Se debe valorar su belleza, utilidad, el gozo de resolver un problema, los retos que propone, entre otros. Sin embargo, la situación actual no cambiará hasta que todos (padres de familia, docentes y estudiantes) modifiquen la visión, en general, que poseen de las matemáticas.

Pero tampoco, como docentes, no se debe pensar que todo está perdido. Romper con un modelo de enseñanza que ha imperado por años y bajo el cual se fue formado es difícil. Hay dos opciones: cruzarse de brazos, ser indiferentes a las necesidades del estudiantado y seguir haciendo lo que se ha hecho durante tanto años; o generar e incorporar, poco a poco, propuestas de cambio en la práctica educativa, orientadas a un "nuevo" tipo de enseñanza acorde con el estudiantado. 
doi: http://dx.doi.org/10.15359/ree.18-2.6

URL: http://www.una.ac.cr/educare

CORREO: educare@una.cr

Los docentes y las docentes debemos tomar consciencia de la necesidad de romper esquemas propios y gastados para lograr modificar los estudiantiles. Esa es nuestra responsabilidad y no podemos esperar que nos digan cómo hacerlo. Al contrario, es nuestro deber buscar estrategias de cambio y compartirlas con nuestros colegas.

Debemos propiciar que la visión de las matemáticas como duras, difíciles y abstractas cambie a una donde se le asocie a afectividad, utilidad e importancia. El reto es difícil, está claro, pero los estudiantes nos lo "reclaman"y debemos responder a ellos.

\section{Referencias}

Akay, H. y Boz, N. (2010). The Effect of Problem Posing Oriented Analyses-II Course on the Attitudes toward Mathematics and Mathematics Self-Efficacy of Elementary Prospective Mathematics Teachers [Actitudes hacia la matemática y autoeficacia en matemáticas de los futuros profesores de matemáticas de primaria]. Australian Journal of Teacher Education, 35(1), 1-75. Recuperado de http://files.eric.ed.gov/fulltext/EJ908190.pdf

Belbase, S. (2010). Images, Anxieties and Attitudes Toward Mathematics [Imágenes, ansiedades y actitudes hacia las matemáticas]. Recuperado de http://files.eric.ed.gov/fulltext/ ED513587.pdf

Benken, B. (2005). Investigating the complexities of mathematics teaching: The Role of Beginning Teachers' Beliefs in Shaping Practice [Investigando las complejidades de la enseñanza de las matemáticas: El papel de las creencias de los docentes principiantes en la conformación de la práctica]. En G. M. Lloyd, M. Wilson, J. L. M Wilkins, \& S. L. Benm (Eds.), Proceedings of the $27^{\text {th }}$ annual meeting for the North American Chapter of the International Group of the Psychology of Mathematics Education, USA, 275. [Actas de la 27 reunión anual para el Capítulo del Grupo Internacional de Psicología de la Educación Matemática de América del Norte]. (pp. 1-6). Recuperado de http://citation.allacademic.com/meta/p mla apa research citation/0/2/4/8/2/p24825 index.html?phpsessid=ui7tgtlc0kv77uc5rvmevv4 4b3

Bernal, A. (2009). Relación de las actitudes de los estudiantes hacia la matemática antes y después de haber cursado y aprobado los programas de Cálculo diferencial e integral en la Universidad Sergio Arboleda (Tesis de Maestría, Universidad Sergio Arboleda). Colombia, Bogotá.

Cadoche, L. y Pastorelli, S. (2005). Concepciones de los alumnos ingresantes a la universidad acerca de la matemática. Revista Premisa, 7(26), 28-34. Recuperado de http://www.soarem. org.ar/Documentos/26\%20Cadoche.pdf

Candia, P. T. (2009). Actitud hacia las matemáticas en alumnos de ingeniería de tercero y quinto semestres del ITESCA. Memoria X Congreso Nacional de Investigación Educativa. Área 5: Educación y Conocimientos Disciplinares. Recuperado de http://www.comie.org.mx/ congreso/memoriaelectronica/v10/pdf/area tematica 05/ponencias/0310-F.pdf 
doi: http://dx.doi.org/10.15359/ree.18-2.6

URL: http://www.una.ac.cr/educare

CORREO: educare@una.cr

Chandía, E., Quiroga, F. y Ulloa, R. (2006). Creencias de los alumnos y profesores de 1 er año de enseñanza media de la intercomuna de Concepción asociadas a la asignatura de matemática. Ponencia presentada en la XIII Jornada de Investigación de Educación Matemática, Concepción, Chile.

Chaves, E., Castillo, M. y Gamboa (abril, 2008). Creencias de los estudiantes en los procesos de aprendizaje de las matemáticas. Cuadernos de Investigación y Formación en Educación Matemática, 3(4), 29-44. Recuperado de http://www.revistas.ucr.ac.cr/index.php/cifem/ article/view/6906/6592

De Faria, E. (2008). Creencias y matemáticas. Cuadernos de Investigación y Formación en Educación Matemática, 3(4), 9-27. Recuperado de http://www.revistas.ucr.ac.cr/index. php/cifem/article/view/6900/6586

Díaz, F. y Hernández, G. (2010). Estrategias docentes para un aprendizaje significativo. México: McGraw-Hill.

Ernest, P. (1988). The Impact of Beliefs on the Teaching of Mathematics [EI impacto de las creencias en la enseñanza de las matemáticas]. En P. Ernest (Ed.), Mathematics Teaching: The State of the Art [Enseñanza de las matemáticas: El estado del arte] (pp. 249-254). London: Falmer Press. Recuperado de http://people.exeter.ac.uk/PErnest/impact.htm

Estrada, M. A. (2002). Análisis de las actitudes y conocimientos estadísticos elementales en la formación del profesorado (Tesis doctoral). Universitat Autónoma de Barcelona. Barceloan, España. Recuperado de http://www.tesisenred.net/bitstream/handle/10803/4697/ maer1de3.pdf?sequence $=1$

Gil, N., Blanco, L. y Guerrero, E. (junio, 2005). El dominio afectivo en el aprendizaje de las matemáticas. Una revisión de sus descriptores básicos. Unión. Revista Iberoamericana de Educación Matemática, 2, 15-32. Recuperado de http://www.fisem.org/www/union/ revistas/2005/2/Union 002 004.pdf

Gil, N., Guerrero, E. y Blanco, L. (enero-abril, 2006). El dominio afectivo en el aprendizaje de las matemáticas. RevistaElectrónica de Investigación Psicoeducativa, 4(1), 47-72. Recuperado de http://www.investigacion-psicopedagogica.org/revista/articulos/8/espannol/Art 896. pdf

Gómez, I. M. (2000). Matemática emocional. Los efectos en el aprendizaje matemático. Madrid: Narcea.

Hernández, G. (febrero, 2011). Estado del arte de creencias y actitudes hacia las matemáticas. Cuadernos de Educación y Desarrollo, 3(24). Recuperado de http://www.eumed.net/rev/ ced/24/ghs.pdf 
doi: http://dx.doi.org/10.15359/ree.18-2.6

URL: http://www.una.ac.cr/educare

CORREO: educare@una.cr

Howe, K. y Berv, J. (2002). Constructing constructivism, Epistemological and Pedagogical [Construyendo el constructivismo epistemológico y pedagógico]. En D. C. Phillips (Ed.), Construtivism in Education: Opinions and Second Opinions on Controversial Issues [Constructivismoeneducación: Opiniónysegundasopiniones sobrecuestionescontrovertidas] (pp. 19-40). Estados Unidos: The Society Margareth Early.

Jensen, E. (2010). Cerebro y aprendizaje. Competencias e implicaciones educativas. Madrid: Narcea Ediciones.

Lamas, H. (enero-diciembre, 2010). Una mirada actual al aprendizaje de las matemáticas. Revista de Psicología, 12(1), 259-328. Recuperado de http://revistas.concytec.gob.pe/pdf/ $\mathrm{rp} / \mathrm{v} 12 \mathrm{n} 1 / \mathrm{a} 12 \mathrm{v} 12 \mathrm{n} 1 . \mathrm{pdf}$

Lazim, M. A., Abu, M. T. y Wan, W. A. (2004). The Statistical Evidence in Describing the Students' Beliefs about Mathematics [La evidencia estadística en la descripción de las creencias de los estudiantes sobre las matemáticas]. International Journal for Mathematics Teaching and Learning, 1-17. Recuperado de http://www.cimt.plymouth.ac.uk/journal/lazimetal.pdf

Liljedahl, P. (2005). Re-Educating Preservice Teachers of Mathematics: Attention to the Affective Domain [Reeducar a futuros profesores de matemáticas: Atención al dominio afectivo]. En G. M. Lloyd, M. Wilson, J. L. M Wilkins, \& S. L. Benm (Eds.), Proceedings of the $27^{\text {th }}$ annual meeting for the North American Chapter of the International Group of the Psychology of Mathematics Education, USA. [Actas de la 27 reunión anual para el Capítulo del Grupo Internacional de Psicología de la Educación Matemática de América del Norte] (pp. 1-7). Recuperado de http://citation.allacademic.com/meta/p mla apa research citation/0/2/4/6/4/p24647 index.html

Martínez, O. J. (2008). Actitudes hacia la matemática. Sapiens, 9(1), 237-256. Recuperado de http://www.redalyc.org/articulo.oa?id=41011135012

McLeod, D. y McLeod, S. (2002). Synthesis-Beliefs and Mathematics Education: Implications For Learning, Teaching, and Research [Síntesis-creencias y educación matemática: Implicaciones para el aprendizaje, enseñanza e investigación]. En G. Leder, E. Pehkonen, \& G. Törner (Eds.), Beliefs: A Hidden Variable in Mathematics Education? [Creencias: Una variable escondida en educación matemática] (pp. 115-123). Londres: Kluwer Academic Publishers.

Mewborn, D. y Cross, D. I. (2007). Mathematics Teachers' Beliefs about Mathematics and Links to Students' Learning [Creencias de los profesores de matemáticas sobre las matemáticas y las relacionadas con el aprendizaje de los estudiantes]. En W. G. Martin, M. E. Strutchens, \& P.C. Elliott (Eds.), The Learning of Mathematics. 69th Yearbook of the National Council of Teachers of Mathematics [El aprendizaje de las Matemáticas. 69 Anuario de la Consejo Nacional de Profesores de Matemáticas] (pp. 259-269). Estados Unidos: Reston, VA. 
doi: http://dx.doi.org/10.15359/ree.18-2.6

URL: http://www.una.ac.cr/educare

CORREO: educare@una.cr

Mora, F. y Barrantes, H. (2008). ¿Qué es matemática? Creencias y concepciones en la enseñanza media costarricense. Cuadernos de Investigacióny Formación en Educación Matemática,3(4), 71-81. Recuperado de http://revistas.ucr.ac.cr/index.php/cifem/article/view/6901/6587

Pantziara, M. y Philippou, G. (2011). Fear of Failure in Mathematics. What are the Sources? [Miedo a las matemáticas ¿Cuáles son las causas? Trabajo presentado en Seventh Congress of the European Society for Research in Mathematics Education University of Rzeszów, Poland. Recuperado de http://www.cerme7.univ.rzeszow.pl/WG/8/CERME\%207 WG8 Pantziara. pdf

Parra, H. (marzo, 2005). Creencias matemáticas y la relación entre actores del contexto. Revista Latinoamericana de Investigación en Matemática Educativa, 8(1), 69-90. Recuperado de http://www.redalyc.org/articulo.oa?id=33508104

Pezzia, M. y Di Martino, P. (2011). The Effect of a Teacher Education Program on Affect: the Case of Teresa and PFCM [El efecto de un programa de formación docente en el afecto: El caso de Teresa y PFCM]. Trabajo presentado en la Seventh Congress of the European Society for Research in Mathematics Education University of Rzeszów, Poland. Recuperado de http:// www.cerme7.univ.rzeszow.pl/WG/8/CERME\%207 WG8 Pezzia.pdf

Ponce, S., Martínez, G. y Zuriaga, F. (2008). Creencias y estereotipos: La dimensión afectiva y su influencia en matemática. Memorias del VI Congreso Argentino de Enseñanza de la Ingeniería (VICAEDI). Ciudad de Salta, Argentina.

Sánchez, D. (2008). Las creencias en la matemática. En Memorias VI Coloquio de Experiencias Educativas en el contexto universitario (pp. 1-12). La Habana: Editorial Universitaria. Recuperado de http://revistas.mes.edu.cu/greenstone/collect/repo/import/ repo/20090319-u/9789591610010028.pdf

Solé, I. (1999). Disponibilidad para el aprendizaje y sentido del aprendizaje. En C. Coll, E. Martín, T. Mauri, M. Miras, J. Onrubia, I. Solé y A. Zabala (Eds.), El constructivismo en el aula (10ª ed., pp. 25-46). Barcelona: Graó.

Solé, I. y Coll, C. (1999). Los profesores y la concepción constructivista. En C. Coll, E. Martín, T. Mauri, M. Miras, J. Onrubia, I. Solé y A. Zabala (Eds.), El constructivismo en el aula (10a ed., pp. 7-23). Barcelona: Graó.

Thompson, A. (1992). Teachers'Beliefs and Conceptions: A Synthesis of the Research [Creencias de los profesores y concepciones: Una síntesis de la investigación]. En D. A. Grouws (Ed.), Handbook of Research in Mathematics Teaching and learning [Manual de investigación en la enseñanza y aprendizaje de las matemáticas] (pp. 127-146). New York, NY: Macmillan. 


\section{(C) Cómo citar este artículo en APA:}

Gamboa, R., (mayo-agosto, 2014). Relación entre la dimensión afectiva y el aprendizaje de las matemáticas. Revista Electrónica Educare, 18(2), 117-139. doi: http://dx.doi.org/10.15359/ree.18-2.6

Nota: Para citar este artículo en otros sistemas puede consultar el hipervínculo "Como citar el artículo" en la barra derecha de nuestro sitio web:

http://www.revistas.una.ac.cr/index.php/EDUCARE/index 